\title{
Ionenwanderung im Gegenstrom als Grundlage für ein elektrochemisches Austauschverfahren
}

\author{
(Isotopentrennung durch Gegenstromelektrolyse)
}

Von Hans Martin

Aus dem Institut für Physikalische Chemie der Universität Kiel

(Z. Naturforschg. 4a, 28-37 [1949]; eingegangen am 22. Dezember 1947)

\begin{abstract}
Es werden Prinzip und theoretische Grundlagen einer elektrolytischen Gegenstrommethode zur Anreicherung von Isotopen mitgeteilt. Der Einzeltrennfaktor $(q)$ ist dureh ein chemisches Austauschgleichgewicht zwischen den Ionen und Neutralteilchen (oder $z$ wischen verschiedenen Ionensorten) eines gelösten isotopen Elektrolyten verursacht. Der die Vervielfachung des Einzeltrenneffekts auf den Trennfaktor $Q\left(=q^{f}\right)$ bewirkende Gegenstrom wird durch Ionenwanderung in einem Flüssigkeitsstrom bewirkt, der so bemessen ist, daß das dem Trennprozeß unterworfene Mischelement keine Überführung erleidet. Die Methode wird an einem Austauschgleichgewicht vom Typus ${ }^{*} \mathrm{II}^{+}+\mathrm{MX} \rightleftarrows \mathrm{M}^{+}+{ }^{*} \mathrm{MX}$, und zwar speziell am Beispiel der Elektrolyse einer $\mathrm{MgSO}_{4}$-Lösung erläutert. Für die mit der Methode erzielbare Vervielfachung $f$ ergibt die Rechnung erwartungsgemäß ungewöhnlich hohe Zahlen. Der Endtrennfaktor $Q_{\mathrm{e}}$ wird ohne und mit Berücksichtigung der Relaxationszeit der Austauschreaktion berechnet. Es ergibt sich, daß $Q_{\mathbf{e}}$ mit wachsender Elektrolysierfeldstärke ein Maximum durchläuft. Das Feldstärkeoptimum gestattet Rückschlüsse auf die Geschwindigkeit der zugrundeliegenden Ionenaustauschreaktion, die hierdurch u.'U. meßbar wird. Die Ergebnisse, die auch hinsichtlich der Erforschung der Natur von Ionengleichgewichten von Interesse sind, werden kurz diskutiert. Uber den zeitlichen Verlauf des Trennvorgangs werden orientierende Angaben gemacht. Zum Schluf folgt eine grundsätzliche Bemerkung zur Frage der Beweglichkeit isotoper Ionen.
\end{abstract}

$\mathrm{D}$ as von U re y und Mitarbb. ${ }^{1}$ entwickelte und in der Folge mannigfach abgewandelte chemische Austauschverfahren zur Anreicherung von Isotopen beruht bekanntlich auf der Tatsache, daß die Gleichgewichtskonstanten für isotope Reaktionsteilnehmer im allgemeinen nicht genau übereinstimmen, und daß daher bei heterogenen chemischen Gleichgewichten, beispielsweise bei $14,15 \mathrm{NH}_{4}^{+}(\mathrm{aq})+\dot{\mathrm{O}} \mathrm{H}-(\mathrm{aq}) \rightleftarrows{ }^{14,15} \mathrm{NH}_{3}(\mathrm{~g})+\mathrm{aq}$, zwischen den beiden Phasen (hier flüssig-gasförmig) hinsichtlich der relativen Isotopenkonzentration Unterschiede auftreten, die sich dann durch die Anwendung der Gegenstrommethode in einer im Prinzip ähnlichen Weise vervielfachen lassen, wie Unterschiede in der Zusammensetzung von Flüssigkeit und Dampf in der Rektifikationssäule. Die beiden Phasen, Isotope in verschiedenem chemischen Bindungszustand enthaltend, bilden dabei gewissermaßen die Führung für die beiden nach dem Gegenstromprinzip geforderten, entgegengesetzt gerichteten Teilchenströme, die ja

1 H. C. U r e y u. L. G r e iff, J. Amer. chem. Soc. 57, 321 [1935]; H. C. Ur ey Rep. Progr. Physics (6. 48 [1940], dort weitere Literatur. auf langem Weg in möglichst enger, rasche Gleichgewichtseinstellung begünstigender Berührung, aber nach dem Bindungszustand der Teilchen wohl getrennt aneinander vorbeizuführen sind.

$$
\text { 1. Das Prinzip }
$$

Die für das Urey sche Austauschverfahren charakteristische Verteilung des Isotopengemischs auf zwei Phasen dient im Grunde genommen einem sekundären Zweck, nämlich der Hervorbringung eines geordneten Gegenstroms. Gelingt es, die gerichteten, den Substanztransport besorgenden Kräfte (in der Regel die Schwerkraft) anstatt an der Phase als Ganzem, individuell an den Teilchen selbst angreifen zu lassen, derart, daß die Atome, die sich im chemischen Gleichgewicht gerade in dem einen Bindungszustand befinden, nach der einen, die Atome, die sich im andern Zustand befinden, in der entgegengesetzten Richtung fortbewegt werden, so muß sich das Gegenstromprinzip auch auf homogene chemische Austauschgleichgewichte anwenden lassen.

Eine Möglichkeit, Teilchengegenströme der so- 
eben gekenmzeichneten Art in einer einzigen Phase mit relativ einfachen experimentellen Hilfsmitteln hervorzurufen, ist nun in der Tat gegeben, nämlich dann, wenn es sich um Gleichgewichte handelt, an welchen Ionen beteiligt sind, denn dann läßt sich ja die Fortbewegung einzelner Teilchen durch ein elektrisches Feld bewirken. Man kann hier zwei Fälle unterscheiden: Im einen ist nur der eine Partner der Austauschreaktion ein Ion, im andern sind beide Partner Ionen, wobei diese dann noch gleiches oder verschiedenes Ladungsvorzeichen haben können. Als Beispiel für den letzteren Fall sei das in einer Cadmiumjodid-Lösung bestehende Gleichgewicht zwischen $\mathrm{Cd}++-$ und $\left[\mathrm{CdJ}_{3}\right]^{--}$Ionen angeführt, welches, als Austauschgleichgewicht geschrieben, ${ }^{*} \mathrm{Cd}+++$ $\left[\mathrm{CdJ}_{3}\right]^{-} \rightleftarrows \mathrm{Cd}+++\left[{ }^{*} \mathrm{CdJ}_{3}\right]-$ lautet $\left(\mathrm{Cd}\right.$ und ${ }^{*} \mathrm{Cd}$ zwei Isotope). Im elektrischen Feld wandern die $\mathrm{Cd}^{++}$-Ionen in der einen Richtung, die $\left[\mathrm{CdJ}_{3}\right]^{--}$ Ionen, Cadmium in anderem Bindungszustand enthaltend, wandern, den Gegenstrom bildend, in der anderen Richtung. Als Beispiel für den ersten Fall können wir die eingangs erwähnte Austauschreaktion zwischen $\mathrm{NH}_{4}+$ und $\mathrm{NH}_{3}$ nehmen, wenn wir sie jetzt als reine Lösungsreaktion formulieren: ${ }^{14} \mathrm{NH}_{4}+(\mathrm{aq})+{ }^{15} \mathrm{NH}_{3}(\mathrm{aq}) \rightleftarrows{ }^{15} \mathrm{NH}_{4}+(\mathrm{aq})$ $+{ }^{14} \mathrm{NH}_{3}(\mathrm{aq})$. Der zum $\mathrm{NH}_{4}+$-Ionen-Hinstrom gehörige Gegenstrom der elektrisch neutralen Teilchen $\left(\mathrm{NH}_{3}\right)$ läßt sich hier, wie im folgenden noch näher erläutert werden soll, durch Aufrechterhaltung eines dem Ionenstrom entgegen gerichteten Flüssigkeitsstroms erzwingen.

Teilchenhin- und -rückstrom vollziehen sich bei solcher Arbeitsweise unter Bedingungen, die eine Annäherung der Reaktionsteilnehmer auf den geringsten überhaupt möglichen Abstand gewährleisten. Wenn man bedenki, daß damit die Einstellzeit des Verteilungsgleichgewichts zwischen Strom und Gegenstrom so kurz wie die Abklingzeit einer Fonenaustauschreaktion wird, so sieht man qualitativ sofort, daß bei der elektrochemischen Austauschmethode - sie sei kurz Gegenstromelektrolyse genannt - gegenüber allen bis-

2 Uber die Theorie der Methode wurde im Dezember 1946 im Physikalisch-chemischen Colloquium der Universität Kiel vorgetragen.

3 A. K. Brew e r, S. L. M a d o r s ky, J. K. T a y lor, V. H. Dibeler, P. B radt, O. L. Par h a m, R. J. B r itte n u. J. G. R e i d jr. J. Res. nat. Bur. Standards 38, 137 [1947]; J. W. We s t h a ve r, daselbst 38, 169 [1947]; S. L. M a d o r s k y u. S. S t r a u s, daselbst 38, 185 [1947]. herigen Anwendungen des Gegenstromprinzips hinsichtlich des Raum- und Zeitbedarfs für eine vorgegebene Vervielfachungswirkung unvergleichlich günstigere Voraussetzungen gegeben sind.

Von solchen Überlegungen ausgehend, haben wir bereits bei Kriegsende die theoretische und - nachdem unter allerdings schwierigsten Arbeitsverhältnissen im Institut die äußeren Voraussetzungen dazu geschaffen waren - gemeinsam mit E. Ruhtz sowie mit W. Jach und E. Vo f die experimentelle Entwicklung der gegenstromelektrolytischen Methode an Hand geeigneter Versuchssubstanzen in Angriff genommen. Über die experimentellen Ergebnisse hoffen wir in Kürze berichten zu können ${ }^{2}$.

Inzwischen ist in Amerika von Brewer, Madorsky, Westhaver und Mitarbb. ${ }^{3}$ über eine Trennung der $\mathrm{K}$ - und der Cl-Isotope durch einen countercurrent electromigration genannten Prozeß berichtet worden, bei welchem die isotopen Ionen gegen einen Flüssigkeitsstrom anwandern, der so bemessen ist, daß das leichter bewegliche leichte Isotop sich gerade noch stromaufwärts, das schwere schon stromabwärts bewegt. Dieses Trennprinzip berührt sich mit dem unseren stärker, als es in Anbetracht der verschiedenen Grundlagen scheinen mag ${ }^{3 a}$; andererseits führt die Diskussion unseres Prinzips, die anschließend kurz durchgeführt werden soll, zu Ergebnissen, die bei allen auf Ionenwanderung fußenden Trennversuchen ${ }^{21,2=}$ grundsätzlich mit in Betracht zu ziehen sind, und daher, namentlich auch nach dem Bekanntwerden der zitierten amerikanischen Arbeiten, von allgemeinerem Interesse sein diirften.

\section{Die Methode}

Zur Vereinfachung der Beschreibung und Diskussion sei den folgenden Erörterungen eine spezielle Versuchssubstanz, und zwar ein binärer

\footnotetext{
3a Aus den Originalarbeiten ist ersichtlich, daß den Ausgangspunkt der amerikanischen Untersuchungen die seit langem bestehende ${ }^{17}$, aber bis dahin nicht bestätigte ${ }^{13-20}$ Vermutung bildet, daß zwei gelöste isotope Ionen im elektrischen Feld verschieden schnell wandern. Die zum Nachweis dieses Effektes benutzte Methode, die zugleich eine kontinuierliche Isotopenanreicherung gestattet, ist im Prinzip mit der unseren identisch. Die bei unseren Überlegungen im Vordergrund stehenden chemischen Austauschgleichgewichte werden auch in den amerikanischen Arbeiten als möglich€ Ursache des Elementareffektes erwähnt, doch wird der Gedanke dort nicht weiter verfolgt. Vgl. auch Anm. 5 a, 12 a, 22 a.
} 
Elektrolyt, zugrunde gelegt. Als solchen wählen wir Magnesiumsulfat.

In einer konzentrierten wäßrigen $\mathrm{MgSO}_{4}$-Lösung werden neben freien (hydratisierten) $\mathrm{Mg}^{++}$-Ionen auch an das $\mathrm{SO}_{4}^{--}$-Ion gebundene, also neutrale Ionendipole oder Moleküle vorliegen, für welche wir kurz $\mathrm{MgSO}_{4} \cdot$ aq schreiben wollen ${ }^{4}$. Wir haben dann die Aus̀tauschreaktion

$$
\begin{aligned}
* \mathrm{Mg}^{++} \cdot \mathrm{aq}+ & \mathrm{MgSO}_{4} \cdot \mathrm{aq} \\
& \rightleftarrows \mathrm{Mg}^{++} \cdot \mathrm{aq}+{ }^{*} \mathrm{MgSO}_{4} \cdot \mathrm{aq},
\end{aligned}
$$

wobei $* \mathrm{Mg}$ etwa das gegen das Hauptisotop ${ }^{24} \mathrm{Mg}$ seltenere ${ }^{26} \mathrm{Mg}$ bedeuten soll. Es kommt nun nach dem eingangs Gesagten darauf an, innerhalb der Lösung einen Magnesium-Kreislauf zu erzeugen, derart, daß das Magnesium dauernd in der Form von $\mathrm{Mg}^{++}$-Ionen von einer Stelle der Lösung nach einer entfernten anderen und von dieser in der Form von undissoziiertem $\mathrm{MgSO}_{4}$ wieder zurück zum Ausgangspunkt transportiert wird. Ein solcher Kreislauf ist mit der in Abb. 1 stark schematisiert angedeuteten Versuchsanordnung und Arbeitsweise im Prinzip leicht realisierbar.

Die Lösung befindet sich in einer elektrolytisehen Zelle, die, abgesehen von erforderlichen, in Abb. 1 fortgelassenen Regelvorrichtungen und Vorrichtungen für die Heran- und Fortschaffung verbrauchter und anfallender Chemikalien, im wesentlichen aus einem längeren Mittelteil, dem Trenn- oder Austauschraum $\mathrm{R}$ (Länge $\mathrm{L}$ ), und aus einem Kathoden- und einem Anodenraum ( $K$ und $A$ ) besteht. Der Austauschraum, der im Prinzip ein dickes Rohr sein könnte, ist in Wirklichkeit in ein Bündel dünner Röhrchen aufgeteilt, die zur Abführung der Stromwärme von außen gekühlt und, zur Vermeidung störender Konvektion, mit feiner Quarzwatte oder dergl. angefüllt werden können.

Bei der gewöhnlichen Elektrolyse würde sich kathodisch $\mathrm{Mg}(\mathrm{OH})_{2}$, anodisch $\mathrm{H}_{2} \mathrm{SO}_{4}$ bilden. Beide Vorgänge lassen sich unterbinden, indem man, wie in Abb. 1 angedeutet ist, in einem der Stromstärke entsprechenden Tempo kontinuierlich der Kathodenflüssigkeit Schwefelsäure, der Anodenflüssigkeit Bariumhydroxydlösung zuführt; durch die Ba-Ionen werden die überschüssigen (d.h. die als $\mathrm{H}_{2} \mathrm{SO}_{4}$ zugeführten) $\mathrm{SO}_{4}$-Tonen als unlösliches $\mathrm{BaSO}_{4}$ aus dem System entfernt. Man sieht, daß auf diese Weise die in der Apparatur vorhandene Gesamtmenge an $\mathrm{MgSO}_{4}$ zeit-

* $\mathrm{Ob}$ es sich hierbei nur um elektrostatische Ionenassoziate $\left(\mathrm{Mg}++\mathrm{aq} \cdot \mathrm{SO}_{4}^{-} \mathrm{aq}\right)$ im Sinne B jerrums (ohne Durchstoßung der Hydrathülle) oder um undissoziierte Moleküle $\left(\mathrm{Mg}++\mathrm{SO}_{4}^{-}\right) \cdot \mathrm{aq}$ handelt, ist vorderhand unwesentlich. Diese Frage wird erst wichtig, wenn man nach der Größe des Isotopieeffektes fragt. lich konstant bleibt. Allerdings würde bei der normalen Elektrolyse das $\mathrm{MgSO}_{4}$ allmählich aus dem Anodenraum in den Kathodenraum überführt werden. Bei der Anordnung der Abb. 1 fließt dem Kathodenraum kontinuierlich Wasser zu, und zwar so schnell, daß die $\mathrm{MgSO}_{4}$-Konzentration in der Kathodenflüssigkeit konstant bleibt; im Anodenraum wird die $\mathrm{MgSO}_{4}$ Konzentration dadurch konstant gehalten, daß aus der Anodenflüssigkeit Wasser im richtigen Tempo kontinuierlich verdampft wird. Auf solche Weise wird in dem Austauschraum $\mathrm{R}$ ein der Wanderungsrichtung der $\mathrm{Mg}++-$ Ionen entgegengesetzt gerichteter Flüssigkeitsstrom erzeugt, der gerade so gro $\beta$ ist, daß im Mittel keine Überführung von Magnesium stattindet. Die mit dem Flüssigkeitsstrom als neutrałe $\mathrm{MgSO}_{4}$-Teilchen schwimmenden Mg-Atome bilden dann einen Teilchenstrom, der dem Teilchenstrom der im elektrischen Feld (durch die entgegenströmende Flüssigkeit

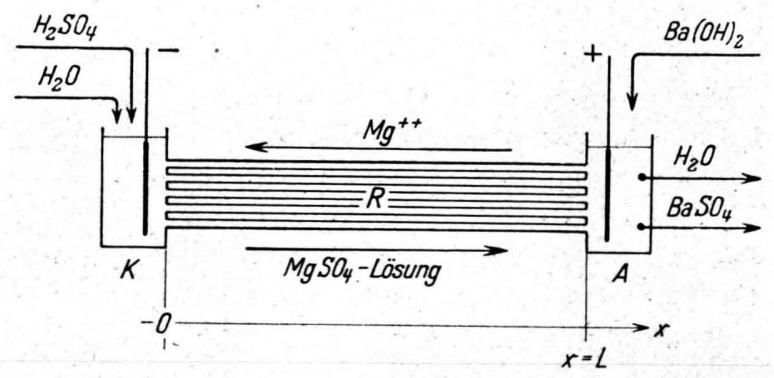

Abb. 1. Prinzip der Gegenstromelektrolyse. Beispiel für eine Versuchsanordnung zur Trennung der Mg-Isotope.

verlangsamt) wandernden freien $\mathrm{Mg}++$-Ionen entgegengesetzt gleich ist ${ }^{5}$. Die Stärke des Flüssigkeitsstromes und damit auch die zu seiner Aufrechterhaltung zu- und abzuführende Wassermenge ergibt sich dabei sofort wie folgt:

Wenn durch den Gesamtquerschnitt $S$ des Trennraums (R) in $1 \mathrm{sec} W \mathrm{~cm}^{3}$ Lösung fließen, so ist die Zahl (in Mol) der Neutralteilchen, die mit diesem Strom pro sec durch $1 \mathrm{~cm}^{2}$ des Querschnitts befördert werden,

$$
\frac{W}{S} c \bar{a}
$$

$\bar{a}$ sei der Assoziationsgrad der Lösung bei der Konzentration $c\left(\mathrm{~mol} \mathrm{~cm}^{-3}\right)$, d. h. der Bruchteil des nicht in freie Ionen dissoziierten Salzes. Die Zahl der im Spannungsgefälle $\Delta P / L$ in umgekehrter Richtung wandernden freien Kationen $\left(\mathrm{Mg}^{++}\right)$

5 Das gilt aueh für den Grenzfall, daß nur freie Ionen vorhanden wären. Hier würden dann die Kationen relativ zur Apparatur gerade stillstehen, wenn man, wie wir es hier tun wollen, davon absieht, daß die isotopen Ionen selbst nicht genau gleich schnell wandern werden (vgl. Schlußkapitel). 
ist dann (in $\mathrm{mol} \mathrm{cm}^{-2} \mathrm{sec}^{-1}$ )

wobei

$$
\left(w_{+}-W / S\right) c(1-\bar{a})
$$

$$
w_{+}=\frac{1}{1-\bar{a}} \frac{\Lambda_{+}}{F} \frac{\Delta P}{L}
$$

die Geschwindigkeit der Kationen in der ruhenden Lösung, $\Lambda_{+}$die Ionen-Äquivalentleitfähigkeit und $F$ die Faradaysche Konstante bedeutet. Gleichsetzen der beiden Teilchenströme liefert, wenn man noch (mittels $\Lambda_{+}=\mathfrak{N}_{+} \Lambda_{c}=\mathfrak{R}_{+} \cdot x / c_{\ddot{\mathrm{A}}}$ ) die Aqquivalentleitfähigkeit $\Lambda_{c}$ des Salzes, die Überführungszahl $\mathfrak{N}_{+}$des Kations, die spezifische Leitfähigkeit $\times\left(\Omega^{-1} \mathrm{~cm}^{-1}\right)$ und die Aquivalentkonzentration $c_{\ddot{\mathrm{A}}}\left(\mathrm{val} \cdot \mathrm{cm}^{-3}\right)$ der Lösung einführt,

$$
\begin{aligned}
W=w_{+}(1-\bar{a}) S=\frac{\Lambda_{+}}{F} \frac{\Delta P}{L} S \\
=\frac{\Lambda_{c} \mathfrak{N}_{+}}{F} \frac{\Delta P}{L} S=\frac{x \Re_{+}}{c_{\ddot{\AA}} F} \frac{\Delta P}{L} S .
\end{aligned}
$$

In dem Prinzipbeispiel der Abb. 1. wird die im Anodenraum anfallende Schwefelsäure durch Ba-Ionen beseitigt. Dazu sei bemerkt, daß natürlich auch andere Arbeitsweisen denkbar sind. So ist es z. B. auch möglich, die Schwefelsäure zu verdampfen, oder, wenn die Vermehrung der Anodenflüssigkeitsmenge nicht stört, sie etwa durch Magnesiumcarbonat, -oxyd oder -metall zu neutralisieren. Auf versuchstechnische Fragen, die im übrigen von Fall zu Fall sehr verschieden sind, soll jedoch hier, wo es nur auf das Prinzipielle ankommt, nicht eingegangen werden.

\section{Der Anreicherungsgrad}

Der theoretisch erzielbare Anreicherungsgrad ist gegeben durch den Einzeltrennfaktor $q$, der hier gleich der Gleichgewichtskonstanten $K$ der Austauschreaktion (1) ist, und durch die Vervielfachung $f$. Wir setzen fest, daß das mit * ausgezeichnete Isotop unter den Neutralteilchen mit dem Molenbruch $\gamma_{2}$, unter den freien Ionen mit dem Molenbruch $\gamma_{+}$vertreten sei, und definieren

$$
q=\frac{\gamma_{\mathrm{a}}}{1-\gamma_{\mathrm{a}}} \mid \frac{\gamma_{+}}{1-\gamma_{+}} .
$$

Ist $q>1$, so reichert sich ${ }^{*} \mathrm{Mg}$ bei der Gegenstromelektrolyse im Anodenraum an. Uber die Höhe der hierbei erzielbaren Vervielfachung gibt dann die folgende Überlegung Auskunft.
Wir fragen nach dem sekundlichen Transport $\dot{n}$ des anzureichernden ausgezeichneten Isotops (in $\mathrm{mol} / \mathrm{sec}$ ), d. h. nach dem Überschuß der den Querschnitt $S$ des Trennraums an der Stelle $x$ in $1 \mathrm{sec}$ in der positiven $x$-Richtung passierenden über die dieselbe Stelle in der úmgekehrten Richtung passierenden Teilchen dieses Isotops. Hierbei setzen wir zunächst voraus, daß das Austauschgleichgewicht durch die Ionenwanderung nicht merklich beeinflußt wird, daß also Gl. (5) an jeder Stelle und zu jeder Zeit gültig bleibt. Dann haben wir, wenn $c_{+}$und $\mathfrak{w}_{+}$die Konzentration bzw. die Translationsgeschwindigkeit der freien Kationen und $c_{a}$ und $\mathfrak{w}_{a}$ die entsprechenden Größen der neutralen Moleküle bedeuten,

$\dot{n}=\gamma_{\mathrm{a}} c_{\mathrm{a}}\left|\mathfrak{w}_{\mathrm{a}}\right| S-\gamma_{+} c_{+}\left|\mathfrak{w}_{+}\right| S-D \frac{d \gamma c}{d x} S$.

Das letzte Glied trägt der Tatsache Rechnung, daß sich im Verlauf des durch die beiden ersten Glieder erfaßten Anreicherungsprozesses in der $x$ Richtung ein Konzentrationsanstieg ausbildet, der eine teilweise Rückdiffusion zur Folge hat. $D$ ist die Diffusionskonstante des Salzes, $\gamma c$ die Konzentration des ausgezeichneten Isotops in der Lösung.

Nun ist der durch das elektrische Feld bewirkte Gesamtstrom an Isotopen $\left(\mathrm{Mg}+{ }^{*} \mathrm{Mg}\right)$ entgegengesetzt gleich dem durch den Flüssigkeitsstrom $W$ bewirkten Isotopenstrom, also

$$
c_{+}\left|\mathfrak{w}_{+}\right| S=c_{\mathbf{a}}\left|\mathfrak{w}_{\mathrm{a}}\right| S=W c_{\mathrm{a}}=W \bar{a} c
$$

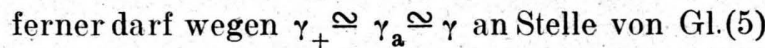

$$
\begin{gathered}
q-1 \simeq \frac{\gamma_{\mathrm{a}}}{\gamma_{+}}-\frac{1-\gamma_{\mathrm{a}}}{1-\gamma_{+}} \quad \text { oder } \\
\gamma_{\mathrm{a}}-\gamma_{+} \simeq(q-1) \gamma_{+}\left(1-\gamma_{+}\right) \simeq(q-1) \gamma(1-\gamma)
\end{gathered}
$$

geschrieben werden. Berücksichtigt man (7) und (8), so lautet die Transportgleichung (6) einfach

$$
\dot{n}=(q-1) W \bar{a} c \gamma(1-\gamma)-D S c \frac{d \gamma}{d x} .
$$

Durch fortgesetzte Elektrolyse wird ein stationärer Endzustand erreicht, in welchem Trennung durch Ionenwanderung und Wiedervermischung durch Diffusion gleich schnell erfolgen, also $\dot{\boldsymbol{u}}=0$ ist. Es gilt dann die Differentialgleichung

$$
(q-1) W \bar{a} \gamma(1-\gamma)-D S \frac{d \gamma}{d x}=0
$$


deren Lösung den gesuchten Gesamttrennfaktor

$$
Q_{\mathrm{e}}=\frac{\gamma /(1-\gamma)}{\gamma_{0} /\left(1-\gamma_{0}\right)}=e^{(q-1) W a L / D S}
$$

liefert, wobei $\gamma_{0}$ und $\gamma$ jetzt die Molenbrüche an den Stellen $x=0$ und $x=L, \mathrm{~d} . \mathrm{h}$. in der Kathodenund Anodenflüssigkeit bedeuten. Wenn wir noch mittels Gl. (4) an Stelle von $W$ wieder elektrische Größen einführen, so erhalten wir schließlich für den Endtrennfaktor $Q_{\mathrm{e}}$ und die Endvervielfachung $f_{\mathrm{e}}$

$$
\begin{aligned}
Q_{\mathrm{e}} & =e^{(q-1) f_{\mathrm{e}}} \sim q^{f_{\mathrm{e}}} \\
f_{\mathrm{e}}=\frac{\Lambda_{+} \bar{a}}{F^{\prime} D} \Delta P & =\frac{\Lambda_{c} \mathfrak{N}_{+} \bar{a}}{F^{\prime} D} \Delta P \\
& =\frac{\varkappa \mathfrak{N}_{+} \bar{a}}{c_{\ddot{\mathrm{A}}} F D} \Delta P
\end{aligned}
$$

Nach Gl. (12) ist der Elektrodenabstand (Trennraumlänge $L$ ) auf die durch Gegenstromelektrolyse mit vorgegebener Spannung $(\Delta P)$ erzielbare Vervielfachung $(f)$ des Austauscheffektes $(q-1)$ ohne Einfluß. Mit der Spannung wächst die Vervielfachung proportional an. Die Gültigkeit dieser Aussage ist an eine wichtige. Voraussetzung geknüpft, die im folgenden besprochen werden soll.

\section{Berücksichtigung der Relaxations- zeit der Austauschreaktion}

Nach den Gln. (11) und (12) sollte der Trennfaktor $Q_{\mathrm{e}}$ mit der angelegten Feldstärke $\Delta P / L$, d.h. mit der Stärke des Teilchenhin- und -rückstroms (Zirkulationsstrom) beliebig anwachsen, während es bekanntlich bei allen anderen Anwendungen des Gegenstromprinzips - Rektifikation, chemisches Austauschverfahren, Clusiussches

5a An Stelle von $\ln Q_{\mathrm{e}}=(q-1) \bar{a} \Lambda_{+} \cdot \Delta P / F D$ (Gl. 12) findet. Westhaver ${ }^{3}$ einen Ausdruck, der in der hier benutzten Schreibweise ähnlich, und zwar $(q-1) \Lambda_{+} \cdot \Delta P / F D$ lautet, worin aber $q$ nicht wie in Gl. (12) eine Gleichgewichtskonstante, sondern das Geschwindigkeitsverhältnis $w_{1} / w_{2}$ zweier isotoper Ionen bedeutet.

5 b Bei der Herleitung von Gl. (12) ist stillschweigend vorausgesetzt, daß die Translationsgeschwindigkeiten $\mathfrak{w}_{\mathfrak{a}}$ und $\mathfrak{w}_{+}$auch quer-zur $x$-Richtung örtlich konstant sind. Das ist aber in praxi nur unvollkommen realisierbar, so daß die experimentellen $f_{\mathrm{e}}$-Werte mehr oder weniger hinter den nach Gl. (12) berechneten zurückbleiben werden. Fragen dieser Art sind in den Arbeiten von Brewer, Madorsky, West haver u. Mitarbb. ${ }^{3}$ experimentell und theoretisch ausführlich behandelt.
Trennrohr, Gegenstrom-Ultrazentrifuge usw. eine optimale Zirkulationsgeschwindigkeit gibt, bei welcher $Q_{\mathrm{e}}$ den größtmöglichen Wert $Q_{\mathrm{e}}{ }^{0}$ annimmt. Der Unterschied ist durchaus charakteristisch, aber er bleibt offenbar nur so lange bestehen, als die bei der Herleitung der Ausdrücke (11) und (12) gemachte Voraussetzung, daß das Austauschgleichgewicht unabhängig von der Ionenwanderung immer vollständig eingestellt sein soll, noch zu Recht besteht. Die Frage, bei welchen Feldstärken unvollständige Gleichgewichtseinstellung zu erwarten ist, soll'im folgenden erörtert werden.

Bei den bisher bekannten Gegenstromverfahren ist die Begrenzung der Zirkulationsgeschwindigkeit nach oben dadurch bedingt, daß der Teilchenaustausch zwischen Strom und Gegenstrom wegen der räumlichen Trennung der beiden Ströme notwendig über eine verhältnismäßig große Entfernung (mittlerer Abstand d) und daher langsam erfolgt. Die Einstelldauer des Verteilungsgleichgewichts (Relaxationszeit $\tau$ ) ist also durch die Dauer $\tau_{\mathrm{D}}$ eines über eine Strecke d erfolgender Diffusionsprozesses gegeben. Nach einer früher mitgeteilten Überlegung ${ }^{6}$ muß nun, wenn der maximal mögliche Trennungsgrad erreicht werden so!l, dafür gesorgt werden, daß ein Teilchen im Strom innerhalb einer Zeit, die der Relaxationszeit $\tau=\tau_{D}$ gleich ist, durch Translation einen Weg zurücklegt, der gerade gleich der Diffusionsstrecke

$$
d=\sqrt{2 D \tau_{\mathrm{D}}}
$$

ist, woraus dann für die optimale Translationsgeschwindigkeit folgt

$$
w^{0}=d / \tau=d / \tau_{\mathrm{D}}=2 D / d=\sqrt{2 D / \tau_{\mathrm{D}}} .
$$

Bei der Gegenstromelektrolyse ist die Relaxationszeit $\tau$ durch die Einstellzeit der chemischen Austauschreaktion gegeben: Um sie zu bestimmen, gehen wir von der Gleichung aus, die man erhält, wenn man die von v. S moluchow ski für die Koagulationsgeschwindigkeit kolloider Teilchen angegebene auf normale bimolekulare Reaktionen ohne Reaktionshemmung anwendet. Nach dieser Gleichung ist die Zahl der Zusammenstöße, die pro sec in $1 \mathrm{~cm}^{3}$ zwischen zwei

${ }^{6}$ H. M a r t in u. W. K u h n, Z. physik. Chem., Abt. A, 189, 219, 317 [1941].

7 M. v. Smoluch ow ski, Z. physik. Chem. 92. 129 [1927]. 
Teilchensorten mit ähnlichen Radien $r$ und Diffusionskonstanten $D$ bei den Konzentrationen $c_{1}$ und $c_{2}$ stattfinden, durch

$$
16 \pi \operatorname{Dr} N_{\mathrm{L}}^{2} c_{1} c_{2}
$$

gegeben. Bei unserer Austauschreaktion treten an Stelle von $c_{1}$ und $c_{2}$ die Konzentrationen $c_{\mathrm{a}}=c \bar{a}$ und $c_{+}=c(1-\bar{a})$ des Mischelements in seinen zwei verschiedenen Bindungszuständen; an Stelle von (15) tritt also

$$
16 \pi D r N_{\mathrm{L}}^{2} c^{2} \bar{a}(1-\bar{a}) \cdot{ }^{8}
$$

Teilen wir durch $N_{\mathrm{L}} c$, die Gesamtzahl der am Austauschgleichgewicht beteiligten Teilchen in $1 \mathrm{~cm}^{3}$, so erhalten wir die Zahl der Stöße, die 1 Atom des Mischelements mit einem im anderen Bindungszustand befindlichen in 1 sec erleidet,

$$
Z=16 \pi D r N_{\mathrm{L}} c \bar{a}(1-\bar{a})
$$

die Zeit, auf welche im Mittel 1 Stoß entfällt, wird

$$
\frac{1}{Z}=\frac{1}{16 \pi D r N_{\mathrm{L}} c \bar{a}(1-\bar{a})}=\tau .
$$

Das ist offenbar die Einstellzeit des Austauschgleichgewichts zwischen Strom und Gegenstrom, vorausgesetzt, daß jeder Sto $\beta$ erfolgreich ist, d. h. zu einem Isotopenaustausch führt.

Während der Zeit $\tau$ erleiden nun die Teilchen nach Gl. (13) durch Diffusion in vorgegebener Richtung im Mittel eine Verschiebung um den Betrag

$$
\lambda=\sqrt{2 D \tau} .
$$

Von dem gleichen Betrag muß nach der Optimumsbedingung (14) die Strecke sein, um die sich bei der Gegenstromelektrolyse ein Ion im elektrischen Feld gegen seine Umgebung, d. h. gegen seinen Reaktionspartner, in der Zeit $\tau$ fortbewegt. Rückdiffusion und Unvollständigkeit der Gleich-

8 Genauer müßte es an Stelle von (15) für verschiedene Teilchen $4 \pi\left(D_{1}+D_{2}\right)\left(r_{1}+r_{2}\right) N_{L}^{2} c_{1} c_{2}$ heißen. Da aber die Diffusionskonstanten der Reaktionsteilnehmer einer Ionenaustauschreaktion wegen der Hydratation im allgemeinen nicht sehr verschieden sein werden, ist es wohl berechtigt, wie in (15) geschehen, mit Mittelwerten $D$ und $r \mathrm{zu}$ rechnen. Für $D$ nehmen wir. einfach den Diffusionskoeffizienten des Salzes, zumal die Berechnung einer „Stoßzahl" bei gelösten Teilchen ohnehin etwas problematisch ist (vgl. A. Eucken, Lehrbuch der Chemischen Physik, Bd. II, 2, S. 1144 f.). gewichtseinstellung werden demnach am günstigsten aufeinander abgestimmt sein, wenn die Wanderungsgeschwindigkeit $w_{+}=\left|\mathfrak{w}_{+}\right|+\left|\mathfrak{w}_{\mathrm{a}}\right|$ gerade den Wert

$$
w_{+}^{0}=\lambda / \tau=\sqrt{2 D / \tau}=4 D \sqrt{2 \pi N_{\mathrm{L}} r c \bar{a}(1-\bar{a})}
$$

hat. Setzt man diesen Ausdruck in die zwischen $v_{+}$und der Feldstärke $\Delta P / L$ bestehende Beziehung

$$
w_{+}=\frac{\Lambda_{c} \mathfrak{N}_{+}}{F} \frac{1}{1-\bar{a}} \frac{\Delta P}{L}
$$

ein, so folgen für die optimale Feldstärke, d.h. für die Feldstärke, bei welcher der Trennfaktor $Q_{\mathrm{e}}$ seinen größtmöglichen Wert $Q_{\mathrm{e}}{ }^{0}$ erreicht, die Ausdrücke

$$
\begin{aligned}
& \left(\frac{\Delta P}{L}\right)^{0}=\frac{F(1-\bar{a})}{\Lambda_{c} \mathfrak{N}_{+}} \sqrt{\frac{2 D}{\tau}}=\frac{4 F D}{\Lambda_{c} \mathfrak{N}_{+}} \\
& \sqrt{2 \pi N_{\mathrm{L}} r c \bar{a}(1-\bar{a})^{3}}=\frac{4 F}{\Lambda_{c} \mathfrak{N}_{+}} \sqrt{\frac{D R T c \bar{a}(1-\bar{a})^{3}}{3 \eta}}
\end{aligned}
$$

deren letzten man erhält, wenn man noch die Zähigkeit $\eta$ des Lösungsmittels mittels des Stokesschen Gesetzes einführt oder an Stelle von (18)

verwendet.

$$
\tau=\frac{3 \eta}{8 k T} \frac{1}{N_{L} c \bar{a}(1-\bar{a})}
$$

Nun verlaufen zwar viele Ionenreaktionen unmeßbar schnell; doch heißt das, da wir uns hier in einem meßtechnisch unzugänglichen Gebiet befinden, keineswegs, daß bei allen diesen Reaktionen praktisch keine, d.h. eine gegen die thermische Energie $(R T)$ vernachlässigbar kleine Aktivierungsenergie $(A)$ vorhanden und somit die Diffusion der Teilchen zueinander der allein geschwindigkeitsbestimmende Vorgang ist.

Wir tragen der Möglichkeit, daß nicht die Diffusion, sondern die eigentliche Reaktion geschwindigkeitsbestimmend ist, in der Weise Rechnung, daß wir in den Gln. (18) bis (21) an Stelle von $\tau$ die größere Relaxationszeit

$$
\tau_{\mathrm{R}}=\frac{1}{\xi} \tau
$$

treten lassen, wobei $\xi$ den Bruchteil der erfolgreichen Stöße bedeutet, der im wesentlichen dureh 
$\exp (-A / R T)$ gegeben ist. Entsprechend ist in Gl. (19) $\lambda$ durch ein größeres

$$
\lambda_{\mathrm{R}}=\sqrt{2 D \tau_{\mathrm{R}}}=\lambda / \sqrt{\xi}
$$

zu ersetzen. An Stelle von Gl. (21) kommt dann für die optimale Feldstärke allgemein

$$
\begin{aligned}
\left(\frac{\Delta P}{L}\right)^{0} & =\frac{F(1-\bar{a})}{\Lambda_{c} \mathfrak{N}} \sqrt{\frac{2 D}{\tau_{\mathrm{R}}}} \\
& =\frac{4 F D}{\Lambda_{c} \mathfrak{N}} \sqrt{2 \pi N_{\mathrm{L}} r c \bar{a}(1-\bar{a})^{3} \xi} \\
& =\frac{4 F}{\Lambda_{c} \mathfrak{N}_{+}} \sqrt{\frac{D R T c \bar{a}(1-\bar{a})^{3}}{3 \eta}} \xi
\end{aligned}
$$

Mit der Herleitung der Ausdrücke (21) und (23) ist die Frage nach dem Gültigkeitsbereich der Gleichungen (11) und (12) beantwortet: Proportionalität zwischen Endvervielfachungswirkung $\left(f_{\mathrm{e}}\right)$ und Elektrolysierspannung $(\Delta P)$ besteht nur, solange

$$
\Delta P \ll L(\Delta P / L)^{0}
$$

bleibt. In der Nähe der Optimalfeldstärke wandern die Ionen so schnell in Gebiete anderer Konzentration $\gamma$ ein, daß sich das Austauschgleichgewicht nurmehr annähernd, aber nicht vollständig nachstellen kann.

Die durch die Gln. (21) und (23) hergestellte quantitative Beziehung zwischen der optimalen Elektrolysierspannung und der Relaxationszeit der Austauschreaktion ist bemerkenswert. Sie gibt im Prinzip die Möglichkeit, die Messung der Relaxationszeit $\left(\tau_{\mathrm{R}}\right)$ von Ionenreaktionen in kon-" densierten Systemen auf die Messung einer optimalen Feldstärke $(\Delta P / L)^{0}$ zurückzuführen und damit die in der Regel extrem hohe Geschwindigkeit solcher Reaktionen u. U. meßbar zu machen. Die Frage freilich, welche von mehreren in Frage kommenden Reaktionen die für einen beobachteten Isotopieeffekt maßgebende ist, wird nicht immer ohne weiteres zu beantworten sein. Hier könnte aber die Tatsache, daß $\tau_{\mathbf{R}}$ und, wie im folgenden noch hervorzuheben sein wird, der Einzeleffekt $(q-1)$ von der Art der von der Reaktion betroffenen Bindungen abhängen, interessante Hinweise liefern.

Der Vollständigkeit halber sei erwähnt, daß sich eine Verallgemeinerung von Gl.(12), die auch im Ge- biet $\Delta P / L \geqq(\Delta P / L)^{0}$ gültig ist, leicht angeben läßt auf Grund der früher in anderem Zusammenhang gefundenen Beziehung ${ }^{9}$

$$
f_{\mathrm{e}}=\frac{2 s}{\boldsymbol{s}^{2}+1} f_{\mathrm{e}}^{0}
$$

durch welche die Endvervielfachung bei optimaler Zirkulationsgeschwindigkeit $\left(f_{\mathrm{e}^{0}}\right)$ mit der bei $s$-facher Optimalgeschwindigkeit $\left(f_{\mathrm{e}}\right)$ verknüpft ist. Unter Benutzung von Gl. (23) findet man so an Stelle von (12) allgemein:

$$
\begin{aligned}
f_{\mathrm{e}} & =\frac{s}{s^{2}+1} \frac{\Lambda_{c} \mathfrak{N}_{+} \bar{a}}{F D}\left(\frac{\Delta P}{L}\right)^{0} L=\frac{2 s}{s^{2}+1} \frac{\bar{a}(1-\bar{a}) L}{\sqrt{2 D \tau_{\mathrm{R}}}} \\
& =\frac{4 s}{s^{2}+1} \bar{a}^{3 / 2}(1-\bar{a}) \quad L\left(\frac{R T c \xi}{3 \eta D}\right)^{1,2}
\end{aligned}
$$

wobei

$$
s \equiv \frac{w}{w^{0}}=\frac{\Delta P / L}{(\Delta P / L)^{0}} .
$$

5. Weitere Diskussion an Hand spezieller Versuchsdaten ( $\mathrm{MgSO}_{4}$-Lösung)

Für die Gegenstromelektrolyse einer 1-m. wäßrigen Magnesiumsulfatlösung mit $100 \mathrm{~V}$ bei $5 \mathrm{~cm}$ Elektrodenabstand ergibt sich nach Gl. (12) in runden Zahlen mit $\Lambda_{c}=50, \mathfrak{N}_{+}=0,25, D=0,55 \cdot 10^{-5}$, $F=96500$

$$
f_{\mathrm{e}}=2300 \bar{a} \text {. }
$$

Als Assoziationsgrad errechnet sich nach $B$ jer$\mathrm{r} \mathrm{u} \mathrm{m}{ }^{10}$ für $c=10^{-3} \mathrm{~mol} \mathrm{~cm}^{-3} \bar{a} \simeq 0,86$, womit $f_{\mathrm{e}} \simeq 2000$ wird. Nun wird der Isotopieeffekt $(q-1)$ in einem Austauschgleichgewicht zwischen Ionen und bloß assoziierten Ionenpaaren zwar außerordentlich gering sein; da aber der $f_{\mathrm{e}}$-Wert ungewöhnlich groß ist, erhält man u. U. trotzdem beobachtbare Trennfaktoren, so hier beispielsweise schon $Q_{\mathrm{e}} \simeq e^{0,2} \simeq 1,2$ für $q=1,0001$, d. h. für den Fall, daß die Gleichgewichtskonstante $K$ der Reaktion (1) nur um $0,1 \%$ von 1 ab. weicht.

Nun werden unter den elektrisch neutralen Teilchen einer Elektrolytlösung neben assoziierten Ionenpaaren (mit einem von Fall zu Fall freilich zwischen 0 oder fast 0 und 1 schwankenden Anteil) auch echte, undissoziierte Moleküle ${ }^{11}$ vertreten sein. Für $\mathrm{MgSO}_{4}$ ergibt sich, wenn man mit dem von C. W. D a vi e ${ }^{12}$ angegebenen Wert der thermodynamischen Konstante des Dissoziationsgleichgewichtes $\left(6,3 \cdot 10^{-3}\right)$ rechnet, daß in der $1-m$. Lösung etwa $1 / 3$ des Salzes undissoziiert

${ }^{9}$ Vgl. H. M a rtin u. W. Kuhn ${ }^{6}$, S. 260 u. 281. 10 N. B j er r u m, Kgl. danske Vidensk. Selsk., math.fysiske Medd. (7) 9 [1929].

11 Vgl. Anm. 4.

12 Vgl. Hand- u. Jahrb. d. Chem. Physik 6, II, W. U l i c h, S. 253; G. K o r t ü m, Elektrolytlösungen, Leipzig 1941, S. 232. 
vorliegen sollte. Rechnete man nur mit echten Molekülen, so wäre $a \simeq 0,33$ statt 0,86 und somit nach (12) auch $f_{\mathrm{e}}$ entsprechend kleiner; trotzdem könnte $Q_{\mathrm{e}}$ größer sein, da im Austauschgleichgewicht undissoziierte Moleküle wesentlich größere Isotopieeffekte $(q-1)$ liefern werden als assoziierte Ionenpaare.

Die vorstehende Untersuchung zeigt, daß die Gegenstromelektrolyse pro Längeneinheit des Trennraums ungewöhnlich hohe Vervielfachungen (f) zu liefern vermag und daß es daher mit Hilfe von handlichen Apparaturen möglich sein wird, auch sehr geringfügige Isotopieeffekte an Ionengleichgewichten meßbar und $u$. U. der praktischen Isotopentrennung nutzbar zu machen ${ }^{12 \mathrm{a}}$. Genauere Messungen solcher Effekte versprechen Aufschlüsse über die Natur der zugrunde liegenden Elektrolytgleichgewichte zu liefern, indem für verschiedene Gleichgewichte (z. B. Ion-Ionenpaar, Ion - Molekül) verschiedene isotopische Spezifität $\mathrm{zu}$ erwarten ist.

Wir wollen für unser obiges Versuchsbeispiel noch die Relaxationszeit $\left(\tau, \tau_{\mathrm{R}}\right)$ und die optimale Feldstärke $(\Delta P / L)^{\circ}$ berechnen. Man wird vermuten, daf Reaktionen vom Typ Gl. (1) praktisch ungehemmt verlaufen, daß also $\xi \approx 1$ ist (Gl.22). Dann findet man mit $\bar{a}=0,86, c=10^{-3}, T=300, \eta=0,01$ nach Gl. (18a) $1,3 \cdot 10^{-9}$ sec und mit den schon angegebenen Zahlenwerten von $D, \Lambda_{c}$ und $\mathfrak{R}_{+}$nach Gl. (21) $10^{5} \mathrm{~V} / \mathrm{cm}$.

Derart hohe Feldstärken lassen sich bei Gleichstromelektrolysen überhaupt nicht realisieren, und man sieht, daß es bei den angenommenen Versuchsdaten $(\Delta P / L=20 \mathrm{~V} / \mathrm{cm})$ tatsächlich berechtigt war, $Q_{\mathrm{e}}$ und $f_{\mathrm{e}}$ nach Gl. (12) und nicht nach Gl. (26) zu berechnen. Es ist aber zu beachten und interessant, daß schon in dem Fall, daß in Wirklichkeit beispielsweise $\xi=10^{-6}$ wäre, die Austauschreaktion also in $\tau_{\mathrm{R}} \approx 10^{-3} \mathrm{sec}$ abliefe - d. i. immer noch unmeßbar schnell -, die Optimalfeldstärke nach Gl. (23) auf $(\Delta P / L)^{0} \simeq 100 \mathrm{~V} / \mathrm{cm}$ absinken und somit in ein experimentell ohne weiteres zugängliches Feldstärkegebiet rücken w ürde ${ }^{13}$.

\section{Geschwindigkeit des Trenn-} prozesses

Die Frage nach dem zeitlichen Verlauf der Gegenstromelektrolyse soll hier nicht näher erörtert wer-

12a B rewer, Madorsky; Westhaver u. Mitarbb. ${ }^{3}$ haben in etwa $10 \mathrm{~cm}$ langen Trennräumen für die Isotope ${ }^{39} \mathrm{~K}$ und ${ }^{41} \mathrm{~K}$ Trennfaktoren $Q \approx 1,5 \mathrm{er}$ zielt.

${ }^{13}$ Niedrige $(\Delta P / L)^{0}$-Werte können nach Gl. (21) auch bei $\xi \approx 1$ auftreten 1 . bei starken Elektrolyten, wenn nur die Bildung echter Moleküle zum Isotopieeffekt beiträgt, indem nach der Elektrolyttheorie in vielen Fällen mit extrem kleinen Assoziationsgraden a zu rechnen ist; 2 . bei schwachen Elektrolyten, indem hier extrem kleine Dissoziationsgrade $1-a$ vorkommen; 3. bei kleinen Elektrolytkonzentrationen $c$. In den, zumal der Verlauf von Gegenstromprozessen im Rahmen der Beschreibung spezieller Trennverfahren (Clusiussches Trennrohr ${ }^{14}$, Ultrazentrifuge ${ }^{6}$ u. a.) in der Literatur ausführlich theoretisch behandelt ist und gewisse hierbei entwickelte Gedankengänge und Resultate allgemeingültig ${ }^{15}$ und leicht auf andere Fälle übertragbar sind. Zur Orientierung seien jedoch einige speziell für das elektrochemische Austauschverfahren gültige Ausdrücke kurz mitgeteilt.

Für den Fall, daß ein seltenes Isotop in kontinuierlichem Betrieb angereichert werden soll, derart, daß unter Konstanthaltung der Ausgangskonzentration $\gamma_{0}$ im einen Elektrodenraum dem anderen laufend das auf $\gamma=Q \gamma_{0}\left(Q \gamma_{0}\right.$ noch $\left.\ll 1\right)$ angereicherte Isotop entnommen wird, ergibt sich (durch Integration von Gl. (9) mit $\dot{n} \neq 0$ und $1-\gamma \cong 1$ ) für den zulässigen Zapfstrom $\dot{n}\left(\mathrm{~mol} \mathrm{sec}^{-1}\right)$ oder für die in $1 \mathrm{sec}$ verarbeitbare Menge Lösung $\dot{V}\left(\mathrm{~cm}^{3} \mathrm{sec}^{-1}\right)$

$$
\begin{aligned}
\frac{\dot{n}}{\gamma_{0} c}=\dot{V} & =(q-1) W \bar{a} \frac{Q_{\mathrm{e}}-Q}{Q_{\mathrm{e}}-1} \\
& =(q-1) \frac{\Lambda_{c} \Re_{+} \bar{a}}{F} \frac{\Delta P}{L} \frac{Q_{\mathrm{e}}-Q}{Q_{\mathrm{e}}-1} S
\end{aligned}
$$

Für das besprochene Versuchsbeispiel ergibt sich so für $S=1 \mathrm{~cm}^{2}$, falls z. B. $q=1,0001, Q_{\mathrm{e}}=1,2$ ist und $Q=1,1$ werden soll, $\dot{V}=10^{-7} \mathrm{~cm}^{3} / \mathrm{sec} \simeq 0,01 \mathrm{~cm}^{3} / \mathrm{d}$, falls $q=1,001, Q_{\mathrm{e}}=7,4$ und $Q=3,7, \dot{V} \simeq 0,1 \mathrm{~cm}^{3} / \mathrm{d}$.

Die Wartezeit für die Ausbildung des Trennfaktors $Q$, die dem Zapfbeginn notwendigerweise vorauszugehen hat, und deren Kenntnis auch bei nicht kontinuierlicher Arbeitsweise wichtig ist, ist, wenn $Q$ seinen Höchstwert $Q_{\mathrm{e}}$ bis auf $\approx 1 / 3$ annähern soll $\left(Q_{\mathrm{e}}-Q\right) /\left(Q_{\mathrm{e}}-1\right) \approx 1 / 3 ;$ Einstellung des stationären Endzustandes zu etwa $2 / 3$ - von der Größenordnung

$$
\Theta \approx \frac{1}{s^{2}+1} \frac{L^{2}}{D} \frac{V_{\mathrm{R}}+V_{\mathrm{A}}}{V_{\mathrm{R}}} .
$$

Für den Fall, daß das Volumen $V_{\mathbf{A}}$ des Vorratsbehälters, in welchem sich das seltene Isotop anreichert, klein gegen das Trennraumvolumen $V_{\mathbf{R}}$ ist, wird in obigem Versuchsbeispiel, wo $s \ll 1, L=5$ und $D=$ $0,55 \cdot 10^{-5}$ war, $\Theta \approx 25$ Tage. Genauer findet man die Annäherung von $Q$ an $Q_{\mathrm{e}}$ mit fortschreitender Zeit $t$ unter Benutzung von Gl. (28) zu

$$
Q=Q_{\mathrm{e}}-\left(Q_{\mathrm{e}}-1\right) \exp \left(-\frac{(q-1) \bar{a} W}{\left(Q_{\mathrm{e}}-1\right) V_{\mathrm{A}}} t\right)
$$

allen drei Fällen wird allerdings nach Gl. (28) auch die Mengenausbeute $(\dot{n})$, in Fall 1 und 2 nach Gl. (12) außerdem der Anreicherungsgrad gering.

$14 \mathrm{Vgl}$. z. B. R. F le i s c hmann u. H. J en se n, Das Trennrohr, Ergebn. exakt. Naturwiss. 20 [1942]; dort weitere Literatur.

15 Besondere diesbezügl. Hinweise z. B. bei: W. Wa lcher, Isotopentrennung. Ergebn. exakt. Naturwiss. 18 [1939]; W. Ku hn u. H. M a r t i n, Z. physik. Chem., Abt. A, 189, 317 [1941]; A. K l e m m, Z. Physik 123, 10 [1944]. 
worin dann $\left(Q_{\mathrm{e}}-1\right) V_{\mathbf{A}} /(q-1) \bar{a} W$ wie $\Theta$ wieder eine Relaxationszeit darstellt.

7. Schlußbetrachtung nebst einer Bemerkung zur Frage der Beweglichkeit is otoper Ionen

Nach der vorliegenden Untersuchung sollte der durch Ionenwanderung im Gegenstrom erzielbare Gesamttrennfaktor wegen der endlichen Einstelldauer des Austauschgleichgewichts mit steigender Elektrolysierfeldstärke $\Delta P / L$ ein Maximum erreichen ${ }^{16}$. Das Feldstärkeoptimum $(\Delta P / L)^{0}$ ist nicht zu erwarten, wenn der Einzeltrenneffekt $(q-1)$, der in jedem Fall auf geringen Unterschieden in den elektrischen Leitfähigkeiten der Isotopen beruht, nicht, wie hier vorausgesetzt ist, ein durch chemische Gleichgewichte bedingter Ionen-Konzentrationseffekt, sondern ein durch unterschiedliche Beweglichkeiten (der freien Ionen) bedingter Ionen-Geschwindigkeitseffekt ist.

Die Frage, ob isotope Ionen (im gleichen chemischen Bindungzustand einschl. Solvatationszustand) in kondensierten Systemen verschiedene Wanderungsgeschwindigkeiten besitzen, ist bereits

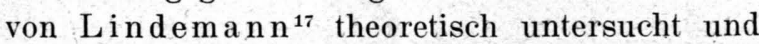
bejahend beantwortet worden. Die Richtigkeit dieser Vorhersage ist später, namentlich unter dem Eindruck zahlreicher negativer Versuche ${ }^{18,19}$, einen solchen Geschwindigkeitseffekt in wäßrigen Lösungen nachzuweisen, wieder in Zweifel gezogen worden, oder man machte (als isotopisch unspezifisch angesehene) chemische Vorgänge, wie Molekülbildung und Hydratation ${ }^{19,}=\mathbf{0}$ der Ionen, für die extreme Geringfügigkeit der an sich nach der kinetischen Theorie der Ionenwanderung erwarteten Masseneffekte verantwortlich. A. K le m m u. Mitarbb. ${ }^{21,22}$, die als erste über erfolgreiche Versuche zum Nachweis eines Wanderungseffektes berichteten, hatten als Versuchssubstanzen $\alpha$-Silberjodid ${ }^{21}$ und eine Lithium-

16 Vgl. demgegenüber A. K l e m m, Z. Physik 123, 10 [1944].

17 A. Lindemann, Proc. Roy. Soc. [London], Ser. A, 99, 102 [1921].

18 J. K e nd a 11 u. Mitarbb., Proc. Nat. Acad. Sci. 9, 75 [1923]; 10, 458 [1924].

19 J. E. G. Pill e y, Philos. Mag. J. Sci. 49, 889 [1925].

20 E. R. J et t e, Philos. Mag. J. Sci. 3, 258 [1927]; J. K e n d a 1 l, Nature [London] 150, 136 [1942].

21 A. K l e m m , Naturwiss. 32, 69 [1944]; Z. Naturforschg. 2a, 9 [1947].

$\because 2$ A. Klem m, H. Hintenberger u. Ph. Hoernes, Z. Naturforschg. 2a, 245. [1947]. chloridschmelze (mit einem KCl-Gehalt) ${ }^{22}$, also nicht-hydratisierte Ionen gewählt. B rew er, M a dorsky, Westhaver u. Mitarbb. ${ }^{3}$, die nunmehr auch über Erfolge bei wäßrigen Lösungen berichtet haben, finden für die hydratisierten Ionen $\mathrm{K}^{+}$und $\mathrm{Cl}-$ Einzeleffekte von einer Größe, die der Herabsetzung des relativen Massenunterschiedes der Ionen durch deren Beladung mit $\mathrm{H}_{2} \mathrm{O}$-Molekeln tatsächlich annähernd entspricht ${ }^{22 a}$.

Zu der Deutung der beobachteten Trenneffekte als Geschwindigkeitseffekte sei nun bemerkt, daß diese Interpretation in jedem Falle so lange als unvollständig (wenn nicht unrichtig) anzusehen ist, wie der Nachweis fehlt, daß Gleichgewichtseffekte nicht im Spiele sind (oder sich gegenseitig kompensieren).

Unter dem Gesichtspunkt von Austauschgleichgewichten würden Isotopieeffekte gerade bei hydratisierten Ionen zu erwarten sein: es genügte, für Isotope verschiedene Hydratationsgrade anzunehmen. Im Falle der zitierten $\mathrm{K}$ lem m schen Versuche ${ }^{22}$ würde die beobachtete anodische Anreicherung von ${ }^{7} \mathrm{Li}$ auf der Basis von Austauschgleichgewichten etwa wie folgt deutbar sein: Angenommen, es fänden sich in der LiCl-Schmelze außer $\mathrm{Li}+$ - und $\mathrm{Cl}$--Ionen z. B. negativ geladene $\left[\mathrm{LiCl}_{x}\right]$-Komplexe; dann.wandern bei der K l e m m schen Versuchsanordnung, bei welcher eine unterschichtete $\mathrm{PbCl}_{2}$-Schmelze (mit Kohleelektrode) Anode ist, außer $\mathrm{Cl}$ - - Ionen auch $\left[\mathrm{LiCl}_{x}\right]$-Komplexe in Richtung auf die stauende Grenzfläche $\mathrm{LiCl}-\mathrm{PbCl}_{2}$. Dort wird der Komplex zerstört (die $\mathrm{Cl}^{--}$-Ionen passieren die Grenze und scheiden aus), die anfallenden $\mathrm{Li}+$ - Ionen wandern, einen Gegenstrom zum [ $\left.\mathrm{LiCl}_{x}\right]$ Strom bildend, zurück. Die Vorgänge tragen also alle Kennzeichen, die wir in Abb. 1 als charakteristisch für die Vorgänge im rechten Teil jenes Gegenstromelektrolyseurs erkannten23,23a. Die Betrachtung läßt sich u. U. auch auf feste Elektrolyte, so auf die von K lemm beobachtete Anreicherung von ${ }^{109} \mathrm{Ag}$ an einer gegen $\alpha$-AgJ gepreßten Kohleanode ${ }^{21}$ ausdehnen:

22a Der Befund steht in Einklang damit, daß die Autoren bei $\mathrm{K}+$ keinen Einfluß des Gegenions $\left(\mathrm{SO}_{4}^{--}, \mathrm{Cl}-\right)$ oder der Salzkonzentration auf den Elementareffekt innerhalb der - für eine sichere Ausschließung von Austauschwirkungen allerdings wohl nicht ausreichenden - Reproduzierbarkeit der Versuche festgestellt haben.

23 Vielleicht kann die von K le mm und Mitarbb. ${ }^{22}$ bei der Schmelzelektrolyse von $\mathrm{LiCl}+\mathrm{KCl}$ gemachte Beobachtung, daß der Elementareffekt, am relativen Massenunterschied gemessen, bei $\mathrm{K}$ größer als bei Li ist, als Hinweis auf die Mitwirkung von Austauscheffekten angesehen werden.

23a Neuerdings hat $\mathrm{A}$. $\mathrm{K} \mathrm{l}$ e $\mathrm{m} \mathrm{m}$ für den elementaren Trennfaktor $q$ einen allgemeinen Ausdruck angegeben, in welchem dem Vorhandensein von Gleichgewichtseffekten neben'dem kinetischen Effekt Rechnung getragen ist (Z. Naturforschg. 3a, 127 [1948]). 
Vom Standpunkt der (hier in Wirklichkeit wandernden) Kohleanode aus betrachtet, haben wir einen ankommenden Teilchenstrom, bestehend aus im AgJGitter fest gebundenen Ag-Ionen, und einen Teilchengegenstrom, bestehend aus den im elektrischen Feld (mehr oder weniger) frei beweglichen Ag-Ionen. Hier würde man freilich weniger von einem chemischen Austauschgleichgewicht als vielmehr von einem Fehlordnungsgleichgewicht im Kristall sprechen.

Eine exakte Vorausberechnung von Austauschgleichgewichten in kondensierten Systemen dürfte allerdings, gleichgültig, ob es sich um Solvatations-, Dissoziations- oder Fehlordnungsgleichgewichte handelt, noch auf erhebliche Schwierig-

\$4 Vgl. H. C. Ur e y, J. chem. Soc. [London] 1947, 562. keiten stoßen ${ }^{24}$. Deshalb wird es vorderhand notwendig sein, die Frage, ob oder inwieweit ein beobachteter Trenneffekt ein Gleichgewichtseffekt (Konzentrationseffekt) oder ein kinetischer Effekt (Geschwindigkeitseffekt) oder beides ist, experimentell durch Variation der Versuchsbedingungen und -substanzen $\mathrm{zu}$ entscheiden. Bei derartigen Untersuchungen wird die Tatsache eine Rolle spielen, daß beim Vorliegen von Austauschgleichgewichten, wie gezeigt wurde, außer der Größe der Austauschkonstanten $K$ die Elektrolytkonzentration $(c, \bar{a})$ sowie $\mathrm{u}$. U. die Trägheit der Gleichgewichtseinstellung (Relaxationszeit $\tau, \tau_{R}$ ) die Größe des Trennfaktors bestimmen.

\title{
Inhomogene Störstellenverteilungen in Trockengleichrichtern
}

\author{
Von Eberhard Spenke \\ Si e m ens-Schuckertwe r ke A.G., Dienststelle Pretzfeld bei Erlangen \\ (Z. Naturforschg. 4a, 37-51 [1949]; eingegangen am 5. Juni 1948)
}

Das Ziel der Arbeit besteht darin, festzustellen, ob beobachtbare Erscheinungen hervorgerufen werden, wenn die Störstellendichte in einem Trockengleichrichter nicht homogen ist, sondern eine Veränderlichkeit mit der Entfernung von der Deckelektrode zeigt.

Zunächst wird in $§ 1$ eine streng gültige Form der Kennliniengleichung abgeleitet, die zu ihrer Auswertung die Kenntnis des Potentialverlaufes quer durch die Randschicht voraussetzt. Ihre Anwendung bietet deshalb manehmal gewisse Vorteile gegenüber der zunächst vorliegenden Form, die die Kenntnis des Verlaufs der Defektelektronen-Konzentration erfordert. Eine aus der streng gültigen Beziehung abgeleitete Gleichungsform

$$
i=\varkappa_{\mathrm{R}} E_{\mathrm{R}}\left(1-e^{-\frac{v}{\mathfrak{B}}}\right)
$$

dürfte für alle praktisch in Frage kommenden Belastungsfälle genügend genaue Resultate ergeben. In $\S 2$ werden Beziehungen zwischen dem Konzentrationsverlauf der Störstellen und der an der Randschicht liegenden Gesamtspannung bzw. der Randfeldstärke $E_{\mathbf{R}}$ abgeleitet, die zur Auswertung von $(1,08)$ erforderlich sind. Neu dürfte aber die Berücksichtigung einer bei ortsveränderlicher Störstellendichte auch im quasineutralen Halbleiterinnern vorhandenen Boltzmann-Feldstärke sein. Mit diesen Mitteln wird im § 3 der Einfluß ortsveränderlicher Störstellendichte bei Sperrbelastungen untersucht. Hier gelingt es, für den differentiellen Widerstand $d U / d i$ einen ähnlich einfachen $\mathrm{Zu}$ sammenhang mit der Störstellenkonzentration herzustellen, wie dies W. Sch ot t k y bereits für die differentielle Kapazität gelungen ist. Leider dürften aber die bei den technischen Gleichrichtern nicht zu vernachlässigenden Feldemissions- und Paßleitungseffekte die Widerstandsbeziehung im Gegensatz zur Kapazitätsbeziehung ungültig machen. Im $\$ 4$ werden einige sprunghafte Störstellenverteilungen bei Flußbelastungen untersucht. Es zeigt sich, daß im Flußgebiet nach der vorliegenden Theorie der differentielle Widerstand praktisch stets exponentiell mit der Flußspannung abnehmen muß, wobei die Abklingspannung gleich dem Temperaturäquivalent $\mathfrak{B}=k T / e$ ist.

$\mathrm{D}^{2}$ ie ursprüngliche Randschichttheorie der Trokkengleichrichter ging von einer Reihe von Hypothesen aus, die in der ersten breiteren Darstellung von W. Schottky $\mathbf{k}^{1}$ ausführlich auf- gezählt worden sind. Daß einige dieser Voraussetzungen bei den technischen Gleichrichtern nur

1 W. Schottky, Z. Physik 113, 367 [1939]; insbes. S. 393 . 